Article

\title{
Experimental Characterization of Unsteady Forces Triggered by Cavitation on a Centrifugal Pump ${ }^{\dagger}$
}

\author{
Dario Valentini ${ }^{1, *}$, Giovanni Pace ${ }^{1}$, Angelo Pasini ${ }^{2}$, Ruzbeh Hadavandi ${ }^{1}$ and \\ Luca d'Agostino ${ }^{2}$ (D) \\ 1 Chemical Propulsion, SITAEL S.p.A., 56121 Pisa, Italy; giovanni.pace@sitael.com (G.P.); \\ ruzbeh.hadavandi@sitael.com (R.H.) \\ 2 Department of Civil and Industrial Engineering, University of Pisa, 56122 Pisa, Italy; \\ angelo.pasini@unipi.it (A.P.); luca.dagostino@ing.unipi.it (L.d.) \\ * Correspondence: dario.valentini@sitael.com; Tel.: +39-050-967-227 \\ $+\quad$ This paper is an extended version of our paper published in Proceedings of the 17th International \\ Symposium on Transport Phenomena and Dynamics of Rotating Machinery (ISROMAC 2017).
}

Received: 6 February 2018; Accepted: 2 May 2018; Published: 7 May 2018

check for updates

\begin{abstract}
The paper presents an experimental campaign aimed at the characterization of the relationship between cavitation-induced instabilities and forces acting on the shaft, relevant to space application turbopumps. The experiments have been carried out on a six-bladed unshrouded centrifugal turbopump. Pressure fluctuations are analyzed in their frequency content for understanding the instability nature (axial, rotating) and their main characteristics (e.g., amplitude, rotating direction). The spectral analysis of the force components highlights a strong relationship of the $z$-component (along the rotating axis) with axial instabilities. On the other hand, rotating cavitation may involve force oscillations along all the three components leading to unwanted and dangerous fluctuating unbalances perpendicular to the rotating axis.
\end{abstract}

Keywords: cavitation; rotordynamic; centrifugal pump; turbomachinery

\section{Introduction}

Propellant feed turbopumps are a crucial component of all liquid propellant rocket engines due to the severe limitations associated with the design of dynamically stable, high power density machines capable of meeting the extremely demanding suction, pumping, and reliability requirements of modern STSs (space transportation systems).

The attainment of such high power/weight ratios is invariably obtained by running the impeller at the maximum allowable speed and low shaft torque. Current configurations in space applications are typically characterized by the presence of lighter, but also more flexible, shafts. Therefore, since the operation under cavitating conditions is tolerated, the turbopump is exposed to the onset of dangerous fluid dynamic and rotordynamic instabilities which may be triggered by cavitation phenomena. When designing a turbomachine, particularly if it has to operate at high rotational speeds, it is important to be able to predict the fluid-induced forces acting on the various components of the turbomachine. The study of radial and rotordynamic forces on turbomachines, by means of analytical/numerical and experimental approaches has been extensively carried out in the last 50 years by many researchers all around the world [1-8]. However, the experimental characterization of the cavitation influence on these phenomena is still very poor even if it is a common operational condition in space application. Moreover, it is extremely important since the occurrence of cavitation drastically modifies the inertia of the fluid surrounding the impellers and, in turn, the critical speeds of the machine. 
Fluid instabilities developing in space inducers have been widely studied in the past [9-17]. However, poor evidences in open literature on experimental activities for centrifugal pumps do not allow a complete understanding of the cavitation induced flow phenomena. In fact, few detailed experimental results can be found in literature focused on the instabilities and their interaction with the system. However, rotating cavitation [18] and auto-oscillation [19] have been detected on centrifugal pumps and studied in previous works.

The experimental characterization of the unsteady fluid forces/moments acting on space turbopump impellers as a consequence of the onset of the most dangerous types of cavitation-induced instabilities is here described and analyzed.

This is done by employing a new methodology, consisting of measuring simultaneously the forces acting on the shaft, by means of a rotating dynamometer previously employed in past experimental activities [20-22], and the pressure field around the centrifugal pump at different locations, by means of piezoelectric pressure transducers. This technique allows for comparing and evaluating the influence of pressure fluctuations on the unsteady force component that the pump exerts on the shaft. The nature of the unsteady flow phenomena interacting with the system can be therefore assessed from the knowledge of mechanical stresses.

\section{Materials and Methods}

\subsection{Experimental Apparatus}

Figure 1 shows the general layout of the cavitating pump rotordynamic test facility (CPRTF, see [23] for further information) at SITAEL S.p.A. (Pisa premise, Italy) (formerly ALTA S.p.A.). The facility is specifically intended for the experimental analysis of the relevant phenomena on space application turbopumps with particular attention on cavitation-related phenomena. The facility operates with water under similarity conditions in order to simulate the behavior of cryogenic fluids [24].

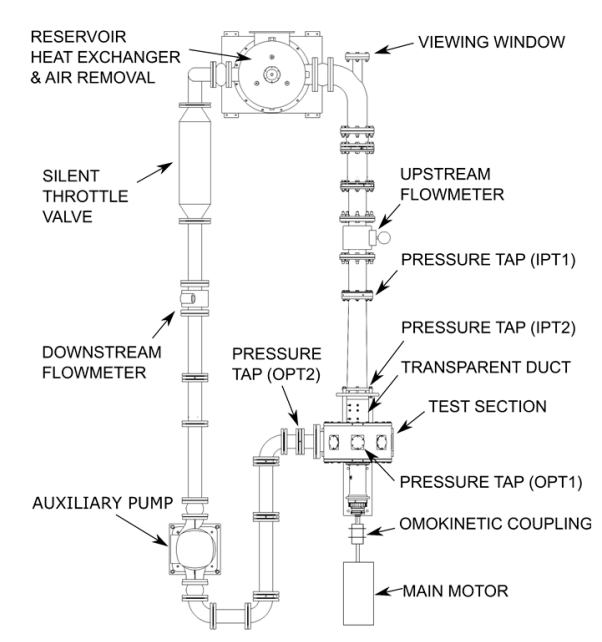

Figure 1. The cavitating pump rotordynamic test facility (CPRTF) layout in SITAEL S.p.A. Abbreviations: OPT1, outlet pressure tap 1; OPT2, outlet pressure tap 2; IPT1, inlet pressure tap 1; IPT2, inlet pressure tap 12 .

In order to relate the fluid instabilities with the unsteady forces acting on the pump, the experiments have been carried out by jointly using a rotating dynamometer (extensively employed in past activities, [25]), and a suitable number of piezoelectric pressure transducers from PCB Piezotronics (Depew, NY, USA), mod. S112A22 3.45 bar range, flush-mounted on the machine casing. Table 1 reports the assessed dynamometer errors with a 95\% confidence level. 
Table 1. Force measurement errors with a confidence level of $95 \%$.

\begin{tabular}{cc}
\hline Force Component & Error (95\% Confidence) \\
\hline$F_{x}$ & $\pm 1.19 \mathrm{~N}$ \\
$F_{y}$ & $\pm 1.49 \mathrm{~N}$ \\
$F_{z}$ & $\pm 1.80 \mathrm{~N}$ \\
\hline
\end{tabular}

The dynamometer has been placed between the pump and the rotor shaft in order to avoid the influence of seals and bearing forces. The positions of the pressure transducers have been chosen in order to evaluate and identify the nature of the fluid instability (rotational/axial), thus different axial and azimuthal positions have been exploited. For this purpose, three different axial positions have been made available for the transducers on the pump casing (Figures 2 and 3): upstream, midstream, and downstream (at the diffuser). With reference to Figure 3, the relative positions of the pressure taps have been designed in such a way that, at the same instant, number-like taps should face the through-flow of the same blade channel. The taps exploited during the present campaign are reported in Table 2.

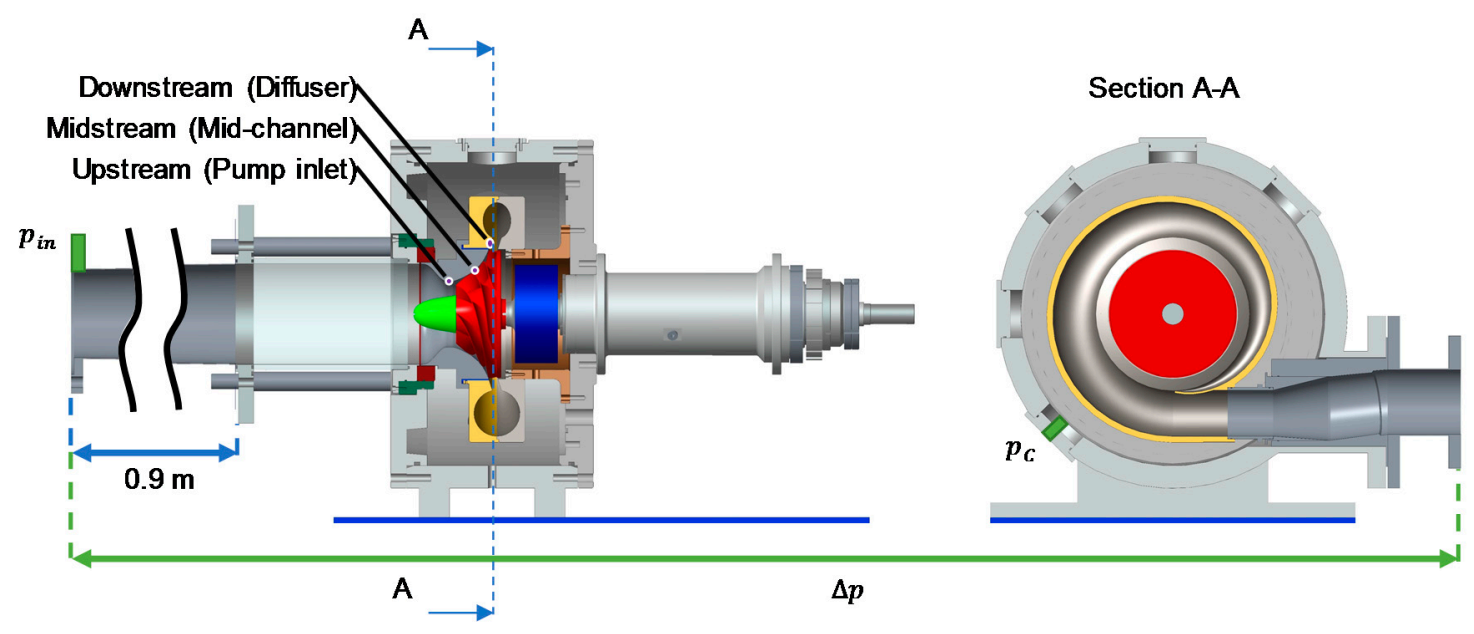

Figure 2. VAMPUFF pump test setup.

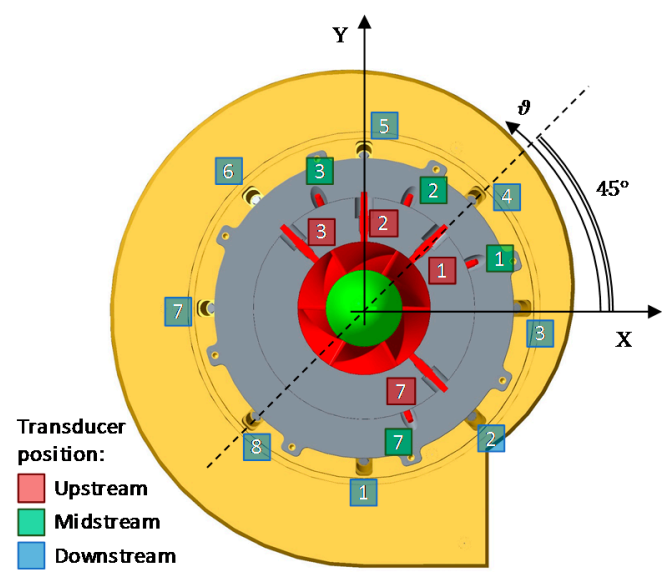

Figure 3. Piezoelectric pressure transducers positions. 
Table 2. Pressure taps exploited for the present campaign.

\begin{tabular}{cc}
\hline Position & Tap \\
\hline Upstream & $1,2,3,4$ \\
Midstream & 1,7 \\
Downstream & $1,2,3,7$ \\
\hline
\end{tabular}

With reference to Figures 1 and 2, the test setup includes:

- two electromagnetic flowmeters (Fisher-Rosemount, Saint Louis, MO, US, model 8732E, range: $0-1001 / \mathrm{s}$, accuracy $0.5 \%$ FS (full scale)), for the measurement of the inlet and outlet flow rates;

- $\quad$ an absolute pressure transducer ( $p_{\text {in }} @$ IPT1) placed six diameters upstream, with respect to the blade leading edge (General Electric, Boston, MA, US, model Druck PMP1400, range 0-1 bar, accuracy $0.25 \% \mathrm{FS}$ ), for the assessment of the inlet cavitation number $(\sigma)$;

- $\quad$ an absolute pressure transducer ( $\left.p_{C} @ O P T 1\right)$ placed in the test chamber/section in order to measure the influence of the pressure on the axial force measured by the dynamometer. In fact, there is a measured force related only to the pressure difference between the water pressure and the sealed dynamometer which is internally at atmospheric condition (General Electric, Boston, MA, US, model UNIK5000, range 0-6 bar, accuracy $0.1 \%$ FS);

- $\quad$ one differential pressure transducer ( $\Delta p @$ IPT1 to OPT2) which measures the pump pressure rise between the inlet station placed at 6 diameters upstream of the blade leading edge, and the outlet station placed about two diameters downstream of the blade trailing edge (General Electric, Boston, MA, US, model UNIK5000, range $0-5$ bar, accuracy $0.1 \%$ FS);

- one temperature sensor PT100 (range $0-100{ }^{\circ} \mathrm{C}$, accuracy $0.5{ }^{\circ} \mathrm{C}$ ).

The test item employed in the present experimental campaign has been designed at SITAEL and is a six-bladed radial pump named VAMPUFF (Figure 4) whose main characteristics are summarized in Table 3. The design is based on the reduced order model developed at SITAEL S.p.A. (formerly ALTA S.p.A.) $[26,27]$ which has been already applied to a similar radial impeller [28]. The clearance between the impeller and the casing has been set to $1 \mathrm{~mm}$.

Table 3. Most relevant geometrical and operational parameters of the centrifugal pump.

\begin{tabular}{|c|c|c|c|}
\hline Parameter & Unit & Symbol & Value \\
\hline Design flow coefficient @ $\mathrm{r}_{2}$ & {$[-]$} & $\Phi_{D}$ & 0.120 \\
\hline Number of blades & {$[-]$} & $N$ & 6 \\
\hline Outlet radius & {$[\mathrm{mm}]$} & $r_{2}$ & 105.00 \\
\hline Inlet tip radius & {$[\mathrm{mm}]$} & $r_{T 1}$ & 55.50 \\
\hline Inlet hub radius & {$[\mathrm{mm}]$} & $r_{H 1}$ & 31.42 \\
\hline Axial length & {$[\mathrm{mm}]$} & $z_{H 2}$ & 67.00 \\
\hline Inlet tip blade angle & [deg] & $\gamma_{T 1}$ & 47.70 \\
\hline Inlet backsweep angle & [deg] & $\chi_{1}$ & 0 \\
\hline Diffuser outlet radius & {$[\mathrm{mm}]$} & $r_{3}$ & 126 \\
\hline Design Rotating Speed & $\begin{array}{c}{[\mathrm{rad} / \mathrm{s}]} \\
{[\mathrm{rpm}]}\end{array}$ & $\Omega$ & $\begin{array}{l}157.1 \\
1500\end{array}$ \\
\hline Design volume flowrate & {$\left[\mathrm{m}^{3} / \mathrm{s}\right]$} & $Q_{\text {des }}$ & 0.022 \\
\hline Mean blade height & {$[\mathrm{mm}]$} & $h_{m}$ & 18.84 \\
\hline Tip solidity & {$[-]$} & $\sigma_{T}$ & 2.64 \\
\hline Incidence tip angle @ design & [deg] & $\alpha$ & 18.90 \\
\hline Outlet tip blade mean angle & [deg] & $\gamma_{T 2}$ & 89.94 \\
\hline Outlet tip backsweep angle & [deg] & $\chi_{2}$ & 64.00 \\
\hline
\end{tabular}




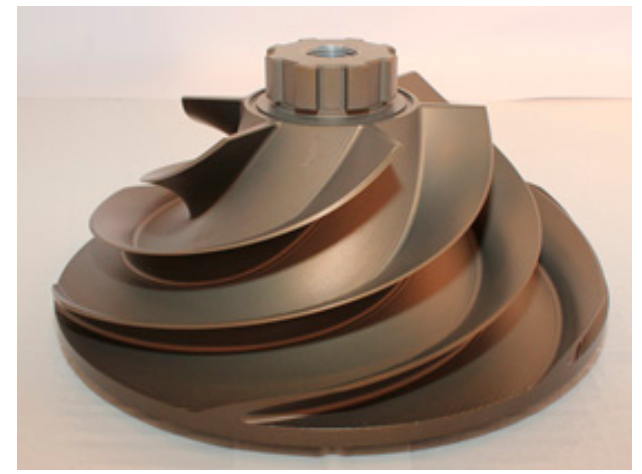

Figure 4. VAMPUFF radial impeller.

\subsection{Pumping Performance}

The tests are performed at room temperature $\left(\mathrm{T} \cong 20^{\circ} \mathrm{C}\right)$, while the inlet pressure is set well above the vapor pressure in order to avoid cavitation inception within the pump. Referring to the pump geometry shown in Table 3, the parameters used to characterize the performance are summarized in Table 4.

Table 4. Pumping performance parameters.

\begin{tabular}{cc}
\hline Total Head Coefficient & $\Psi_{\mathrm{T}}=\Delta p_{T} / \rho \Omega^{2} r_{2}^{2}$ \\
Flow Coefficient & $\Phi=Q / \Omega r_{2}^{3}$ \\
Reynolds Number & $R e=2 \Omega r_{2}^{2} / v$ \\
\hline
\end{tabular}

\subsection{Cavitating Performance}

Steady-state cavitating experiments are performed by maintaining a fixed inlet pressure condition during the tests. Continuous cavitating experiments are performed by linearly lowering the inlet pressure in order to reach different cavitating regimes; the pressure decrease rate is approximately $0.003 \mathrm{bar} / \mathrm{s}$. Signals are acquired at 5000 samples per second and each continuous experiment is divided into sub-blocks of $5 \mathrm{~s}$ in order to allow a quasi-steady hypothesis as experimentally demonstrated in previous works $[17,29]$ and highlighted in the following results. Per each sub-block the operating conditions and relevant phenomena do not change significantly. The correspondent frequency resolution $(\Delta f)$ is $0.2 \mathrm{~Hz}$. Moreover, each sub-block is superposed with the following one by $50 \%$ in order to catch all the phenomena. The main parameters used to characterize the cavitating performance are in Table 5.

Table 5. Cavitating performance parameters.

\begin{tabular}{cc}
\hline Head Coefficient & $\Psi=\Delta p / \rho \Omega^{2} r_{2}^{2}$ \\
Cavitation Number & $\sigma=\left(p_{\text {in }}-p_{v}\right) / 0.5 \rho \Omega^{2} r_{2}^{2}$ \\
\hline
\end{tabular}

\subsection{Flow Instabilities and Unsteady Fluid Forces}

The pressure fluctuations of the flow through the pump triggered or not by cavitation have been investigated during the cavitating experiments. Cervone et al. [16], and Pace et al. [29] report the procedures already exploited by the authors and summarized here. As for the cavitating performance evaluation, the signals, acquired by means of the piezoelectric transducers located on the casing of the pump and the diffuser, are divided into sub-blocks. Per each sub-block, the signals are fast-Fourier transformed in order to obtain the frequency content of oscillating phenomena. At each frequency of interest, the analysis of the cross-spectrum phase of signals from transducers placed at the 
same axial location but at different azimuthal positions allows for understanding the physical nature of the phenomenon. In fact, oscillating axial phenomena involve signals characterized by zero-phase cross-spectrum. On the other hand, rotating phenomena lead to cross-spectrum phases $(\varphi)$ proportional to the circumferential spacing $\Delta \vartheta$ between transducers and proportional to the phenomenon number of lobes $(n)$. For instance, for a generic cross-spectrum from the transducer 1 to the transducer 2, it would be

$$
n=\left|\varphi_{12} /\left(\vartheta_{2}-\vartheta_{1}\right)\right|,
$$

where negative values of $\varphi_{12}$ imply a phenomenon moving from transducer 1 to transducer 2 . Aliasing in the azimuthal direction is eliminated by comparison of the cross-correlations of pressure signals from transducers with different angular separations. The analysis is validated based on the value of the coherence function between signals obtained from two generic transducers $(x, y)$ $\gamma_{x y}=\left|S_{x y}(f)\right|^{2} /\left[S_{x x}(f) S_{y y}(f)\right]$, where $S$ is either the cross-spectrum or the auto-spectrum of the referred transducers $(x, y)$ in the subscripts (see [30] for further information). In the present work, only phenomena with values of $\gamma_{x y}$ greater than 0.95 have been considered.

In order to understand the frequency content of the signals recorded through the dynamometer, it is useful to consider and compare the effect of different types of unsteady forces on the dynamometer rotating frame ( $x y z$ ) rotating with speed $\Omega$ and on the fixed one $(X Y Z)$, where $z$ and $Z$ are directed along the rotating axis. Therefore, the force evaluated in the two reference frames has the same $z$-component as well as the same total force acting on the plane $\mathrm{X}-\mathrm{Y}(x-y)$.

Considering Figure 5, the schematic proposed involves a fluctuating phenomenon generating a force with constant direction $\left(\theta_{0}\right)$ with respect to the fixed reference frame, a mean value $F_{0}$ and an oscillating amplitude: $F_{1}=F_{0}+\widetilde{F}_{1} \cos \left(\omega_{2} t\right)$. Furthermore, the schematic considers a constant amplitude force $F_{2}$ rotating with respect to the fixed reference frame with an angular velocity $\omega_{1}$. The force components in the rotating frame and in the fixed one are

$$
\begin{gathered}
F_{X}=F_{0} \cos \left(\theta_{0}\right)+\widetilde{F}_{1} \cos \left(\omega_{2} t\right) \cos \left(\theta_{0}\right)+F_{2} \cos \left(\omega_{1} t\right), \\
F_{Y}=F_{0} \sin \left(\theta_{0}\right)+\widetilde{F}_{1} \cos \left(\omega_{2} t\right) \sin \left(\theta_{0}\right)+F_{2} \sin \left(\omega_{1} t\right), \\
F_{x}=\frac{\widetilde{F}_{1}}{2} \cos \left[\left(\omega_{2}-\Omega\right) t+\theta_{0}\right]+\frac{\widetilde{F}_{1}}{2} \cos \left[\left(\omega_{2}+\Omega\right) t-\theta_{0}\right]+F_{2} \cos \left[\left(\omega_{1}-\Omega\right) t\right]+F_{0} \cos \left(\Omega t-\theta_{0}\right), \\
F_{y}=\frac{\widetilde{F}_{1}}{2} \sin \left[\left(\omega_{2}-\Omega\right) t+\theta_{0}\right]-\frac{\widetilde{F}_{1}}{2} \sin \left[\left(\omega_{2}+\Omega\right) t-\theta_{0}\right]+F_{2} \sin \left[\left(\omega_{1}-\Omega\right) t\right]-F_{0} \sin \left(\Omega t-\theta_{0}\right) .
\end{gathered}
$$

Therefore, a frequency analysis of the force components would show two frequencies $\left(\omega_{1} / 2 \pi\right.$, $\left.\omega_{2} / 2 \pi\right)$ in the fixed reference frame. On the other hand, the same analysis in the rotating frame would show multiple frequencies shifted from the above-mentioned ones by $\pm \Omega / 2 \pi$.

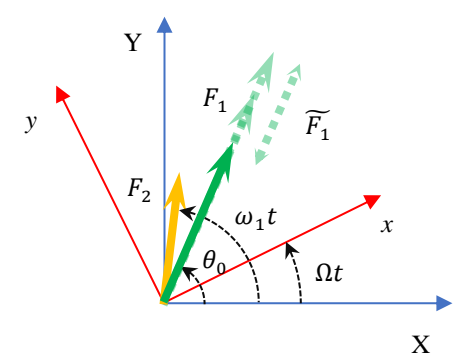

Figure 5. Schematic of a generic unsteady force acting on the shaft with respect to the rotating frame $(x y)$ and the fixed one $(X Y) . F_{1}$, force with a fixed direction $\theta_{0}$ and oscillating amplitude; $F_{2}$, rotating force with velocity $\omega_{1} ; \Omega$, rotating frame speed. 


\section{Results and Discussion}

\subsection{Pumping Performance}

Figure 6 shows the pumping performance obtained at three different rotating speeds. The data overlapping confirms the Reynolds independence of the tests (fully turbulent flow).

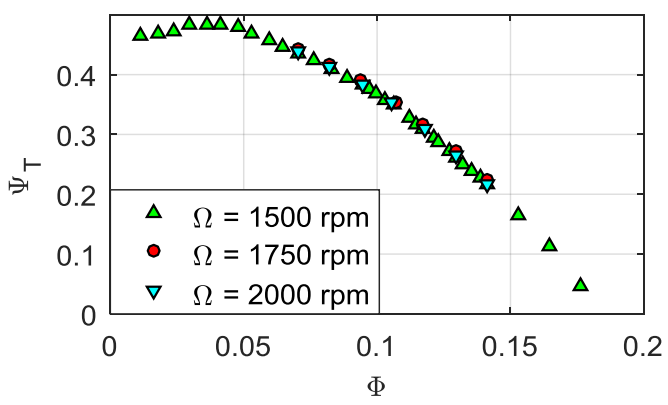

Figure 6. VAMPUFF total head $\left(\Psi_{T}\right)$ versus flow coefficient $(\Phi)$ at different rotating speed $(\Omega), \mathrm{T} \cong 20^{\circ} \mathrm{C}$.

\subsection{Cavitating Performance}

Figure 7 reports the VAMPUFF pump cavitating performance obtained at different flow coefficients $\Phi$. Per each flow coefficient, solid markers with black borders show results obtained from steady-state experiments, while blank markers report continuous experiments results per each sub-block. The overlapping of the data from the two different procedures confirms the goodness of the continuous data and the steady-state hypothesis which can reliably represent the cavitating performance. The rotational speed $\Omega 1750 \mathrm{rpm}(183.3 \mathrm{rad} / \mathrm{s}, 29.2 \mathrm{~Hz})$ prevent the exceeding of the maximum force measure capability of the dynamometer, allowing at the same time to obtain relevant cavitation phenomena. Other relevant parameters are reported in Table 6.

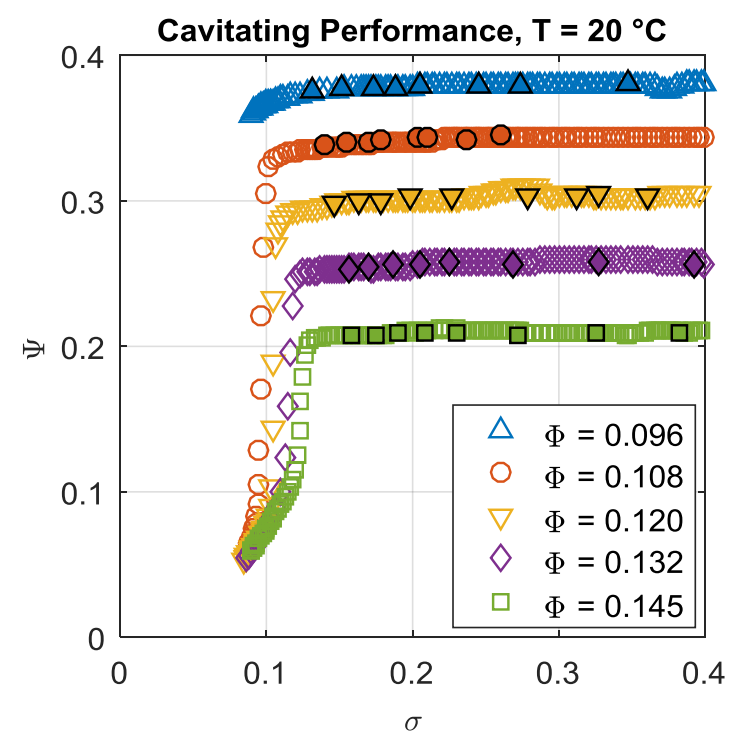

Figure 7. VAMPUFF cavitating performance as head coefficient $(\Psi)$ versus cavitation number $(\sigma)$ at different flow coefficients $(\Phi)\left(\mathrm{T}=20^{\circ} \mathrm{C} ; \Omega=1750 \mathrm{rpm}\right)$. 
Table 6. Relevant parameters for the cavitating experiments.

\begin{tabular}{cc}
\hline Sampling Frequency $\left(f_{s}\right)$ & $5000 \mathrm{~Hz}$ \\
Temperature & $20{ }^{\circ} \mathrm{C}$ \\
Experiment Duration (cont.) & $240 \mathrm{~s}$ \\
Sub-Block Duration & $5 \mathrm{~s}$ \\
\hline
\end{tabular}

\subsection{Flow Instabilities and Unsteady Fluid Forces}

The systematic evaluation of the flow instabilities during the cavitating experiments highlighted the presence of rotating and axial phenomena for different flowrates, as briefly summarized in Table 7 . In the table, at the same flowrate, oscillating phenomena showing analogous characteristics (rotating with 1 lobe, axial, etc.) are grouped together regardless of the operating regimes ( $\sigma$, frequency).

Table 7. Identified phenomena at different flowrates.

\begin{tabular}{cc}
\hline $\boldsymbol{\Phi}\left(\boldsymbol{\Phi} / \boldsymbol{\Phi}_{\boldsymbol{D}}\right)$ & Identified Phenomena \\
\hline $0.096(0.8)$ & Rotating (1 lobe and 2 lobes); axial \\
$0.108(0.9)$ & Rotating (1 lobe and 2 lobes); axial \\
$0.120(1.0)$ & Rotating (1 lobe); axial \\
$0.132(1.1)$ & Rotating (1 lobe); axial \\
$0.144(1.2)$ & Axial \\
\hline
\end{tabular}

The presence of cavitation instabilities leads to unwanted forces on the shaft even at $\Phi=\Phi_{D}$. However, at design condition the intensity of the detected oscillating forces is minor than at lower flowrates. For this reason, the present study only focuses on $\Phi=0.108\left(\Phi / \Phi_{D}=0.9\right)$ in order to show some of the typical pressure-force spectra relationships found at all the tested regimes.

Figure 8 reports the cavitating performance at a nominal $\Phi_{N}=0.108$ as well as the flow coefficient behavior during the inlet pressure decay. At the end of the experiment, the massive presence of cavitation leads to the breakdown which, in turn, leads to the flow coefficient drop.

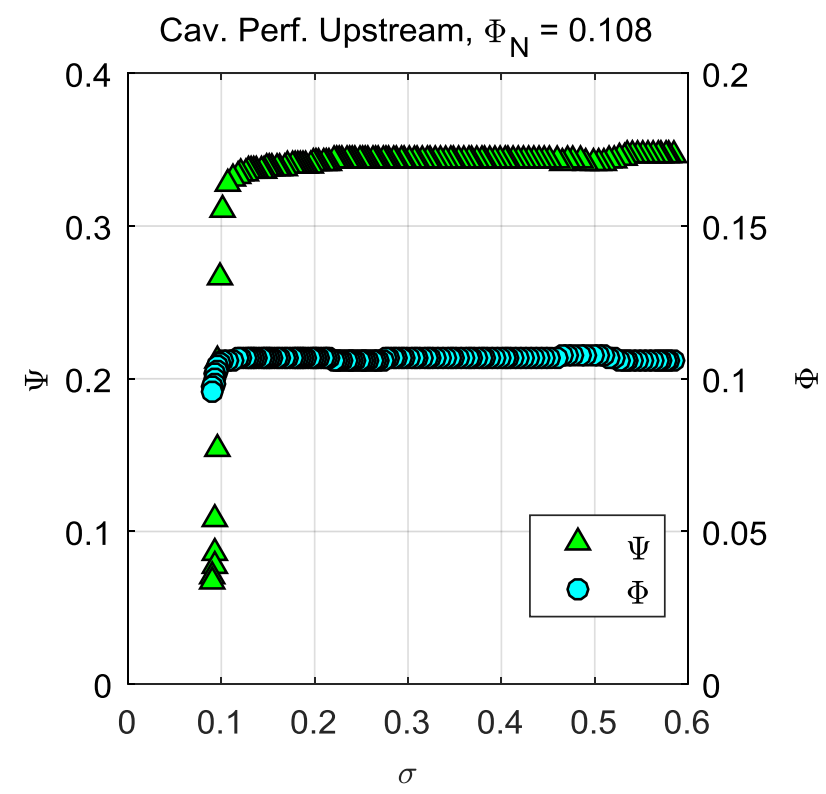

Figure 8. Cavitating performance (head coefficient $\Psi$ versus flow coefficient $\Phi$ ) and flow coefficient evolution, nominal $\Phi_{N}=0.108\left(90 \% \Phi_{D}\right)$. 
The flow instabilities illustrated in this paper are basically connected with the presence of cavitation. According to [31], cavitation inception usually starts in the tip vortex generated at the blade inlet. Therefore, it is of no surprise that the major part of the found phenomena is clearly visible by the upstream pressure transducers, becoming less visible while moving downstream. On the other hand, axial phenomena effectively propagate from the upstream to the downstream and vice versa, becoming clearly visible in both stations. In the figures, $\Omega$ is intended as the rotating frequency.

Figure 9 reports the energy frequency content of three pressure transducers as representative of the three different stream locations: upstream, midstream, and downstream (according to Figures 2 and 3) versus the cavitation number $\sigma$. The frequency energy content $\left(E_{f}\right)$ is directly related to the amplitude $\left(A_{f}\right)$ of the acting oscillating phenomenon as follows:

$$
E_{f}=\frac{A_{f}^{2}}{4} \frac{N_{s}}{f_{s}}
$$

where $N_{s}$ is the number of samples considered for the fast Fourier transform (FFT) while $f_{s}$ is the sampling frequency exploited during the experiment.

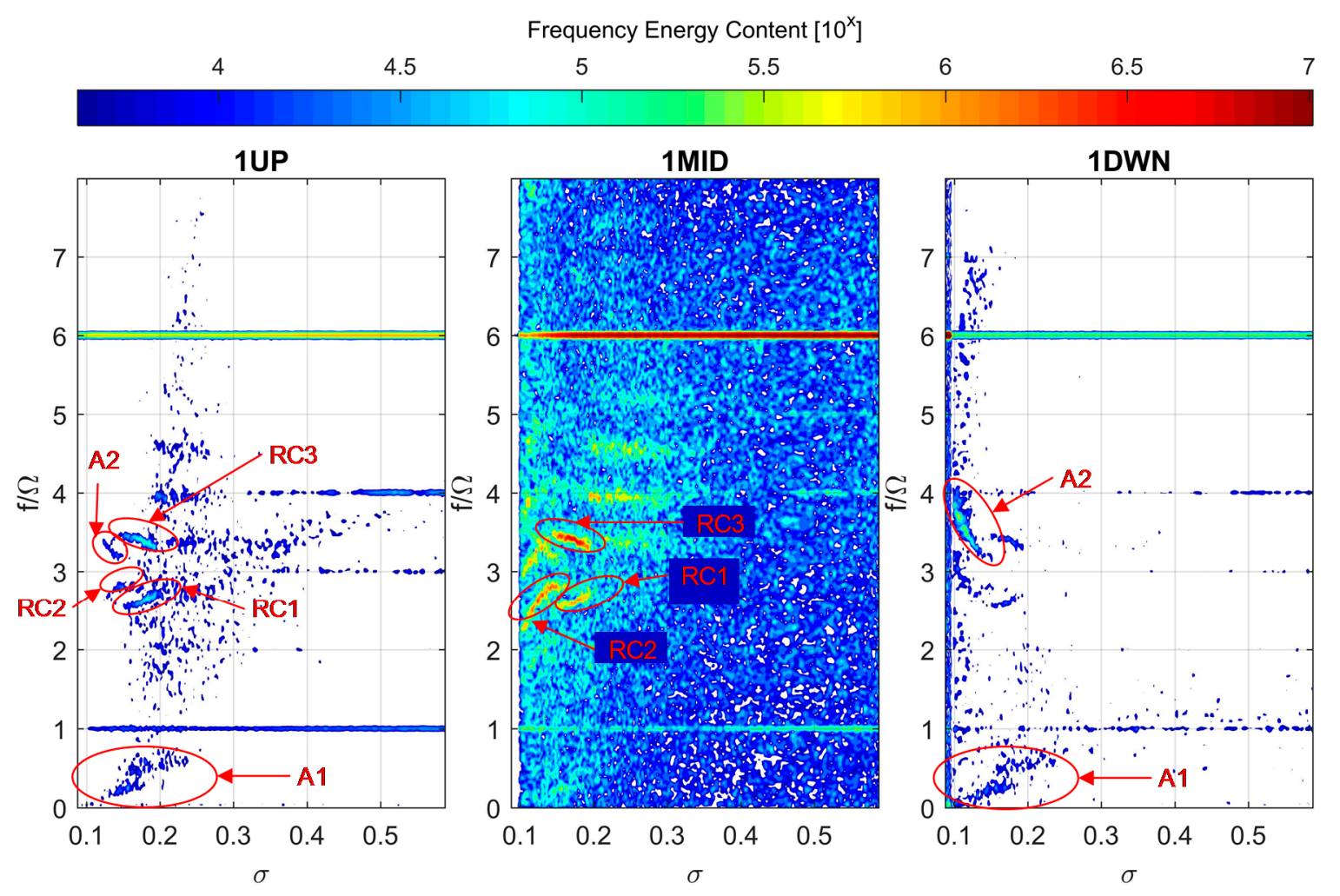

Figure 9. Frequency energy content $\left[\mathrm{Pa}^{2} \cdot \mathrm{s}\right]$ of pressure transducers placed upstream (left), midstream (center), and downstream (right). The red circled identified phenomena are reported in Table 8. Abbreviations: UP, upstream; MID, midstream; DWN, downstream; RC, rotating cavitation; A, axial.

While the blade passage frequency is clearly visible at $6 \Omega$ per each station, the other relevant phenomena are generally of major interest at a single station. In order to understand the physical nature of such oscillating phenomena, Figures 10-12 report the phase of the cross-spectrum of the upstream, midstream, and downstream pressure transducers, respectively. The figures show only the phenomena of major interest characterized by an amplitude $A_{f} \geq 100 \mathrm{~Pa}$ and a coherence $\gamma_{x y} \geq 0.95$, focusing on the range of $f / \Omega=[0,4]$ where interesting phenomena have been found. The phase 
values allow for understanding the nature of the phenomenon [16,17]. The main outcomes of this analysis are summarized in Table 8.

Table 8. Summary of the found instabilities at $\Phi_{N}=0.108$. Abbreviations: RC, rotating cavitation; A, axial.

\begin{tabular}{ccccc}
\hline ID & $\begin{array}{c}\text { Frequency Range } f / \Omega \\
(f[\mathrm{~Hz}])\end{array}$ & $\sigma$ Range & Characteristics & Major Station \\
\hline A1 & $\begin{array}{c}0.1-0.38 \\
(3-11)\end{array}$ & $0.16-0.12$ & Axial & $\mathrm{Up}$ \\
RC1 & $\begin{array}{c}2.57-2.74 \\
(75-80)\end{array}$ & $0.2-0.16$ & Rotating 1 lobe & $\mathrm{Up}$ \\
$\mathrm{RC} 2$ & $\begin{array}{c}2.33-2.85 \\
(68-83)\end{array}$ & $0.16-0.11$ & Rotating 2 lobes & Mid \\
A2 & $\begin{array}{c}3.22-3.81 \\
(94-111)\end{array}$ & $0.16-0.10$ & Axial & Down \\
RC3 & $\begin{array}{c}3.26-3.46 \\
(95-101)\end{array}$ & $0.19-0.14$ & Rotating 1 lobe & Mid \\
\hline
\end{tabular}

$\begin{array}{lllllll}-150 & -100 & -50 & 0 & 50 & 100 & 150\end{array}$
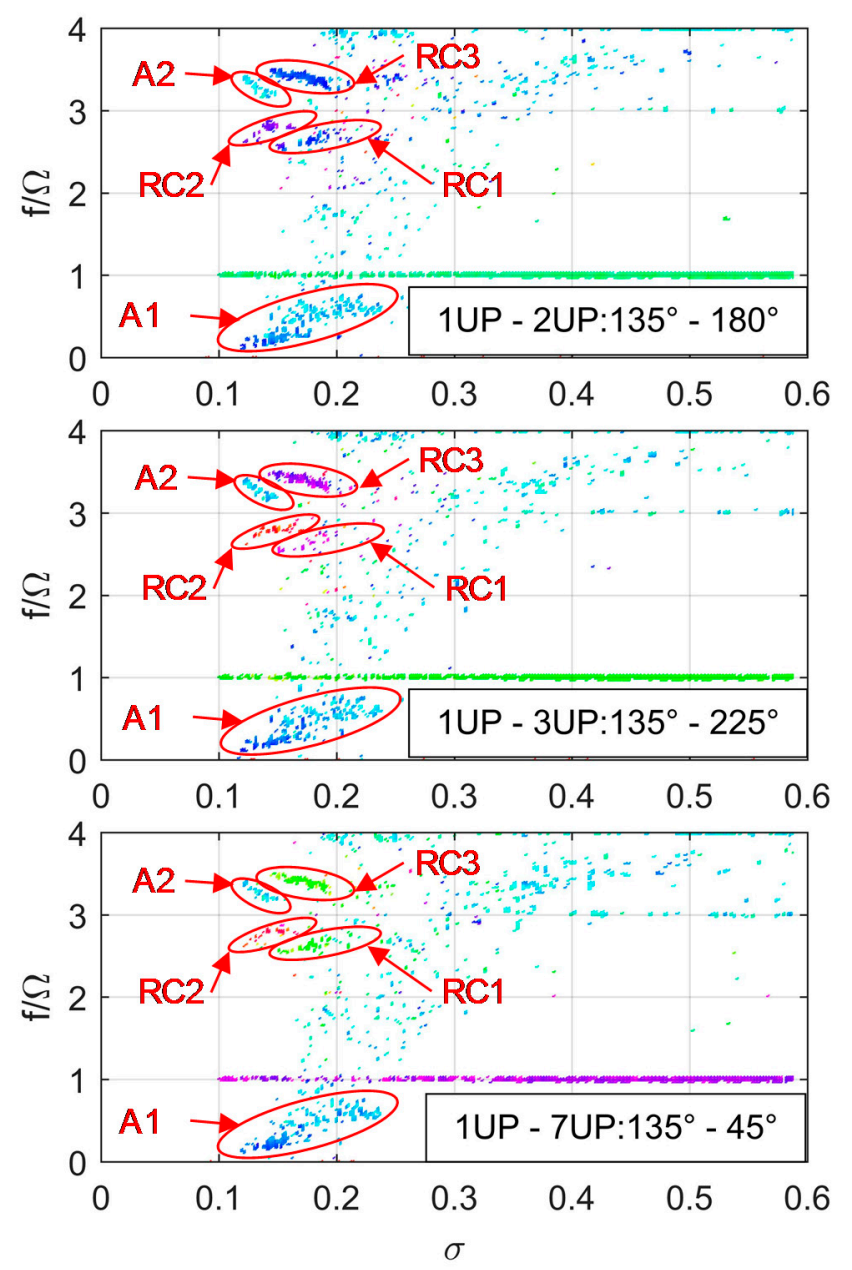

Figure 10. Cross-spectrum phase $(\varphi)$ of the upstream pressure transducers, $\Phi=0.108\left(90 \% \Phi_{D}\right)$. Red circled phenomena are summarized in Table 8. 


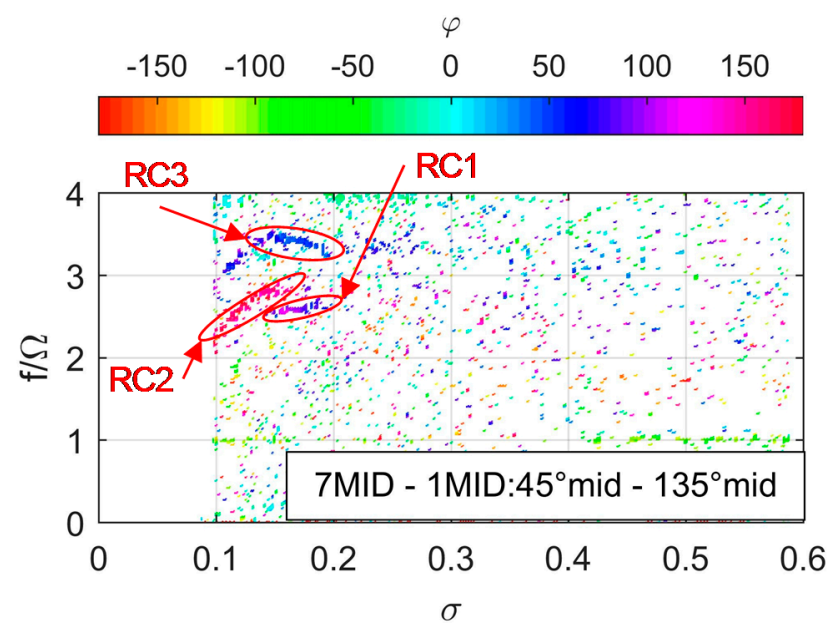

Figure 11. Cross-spectrum phase $(\varphi)$ of the midstream pressure transducers, $\Phi=0.108\left(90 \% \Phi_{D}\right)$. circled phenomena are summarized in Table 8.
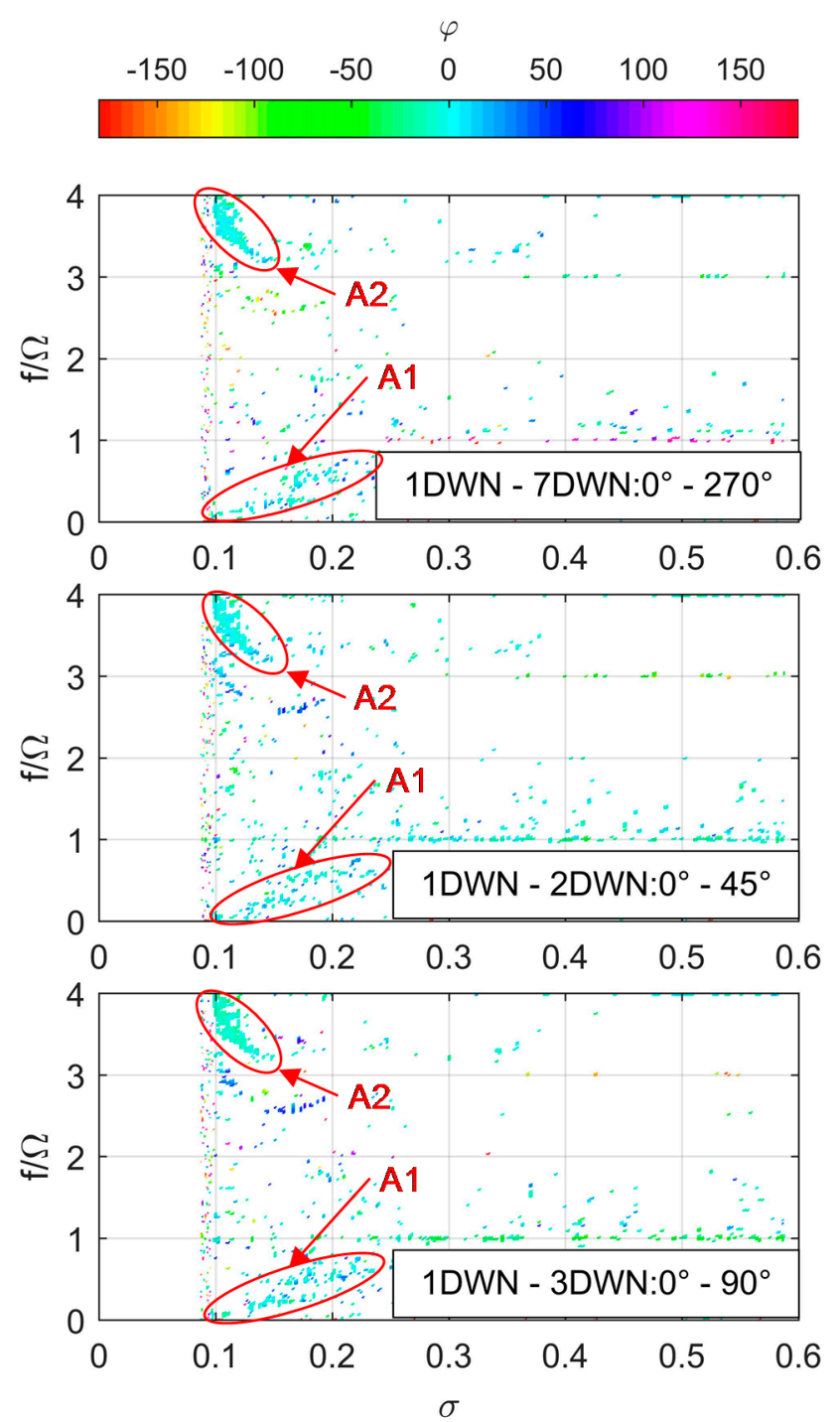

Figure 12. Cross-spectrum phase $(\varphi)$ of the downstream pressure transducers, $\Phi=0.108\left(90 \% \Phi_{D}\right)$. Red circled phenomena are summarized in Table 8. 
Figure 13 shows the frequency energy content of the force components measured by the dynamometer with a force amplitude oscillation greater than $0.5 \mathrm{~N}$. In particular, the figure reports the force components independent of the chosen reference frame (i.e., rotating or fixed), as described previously.

$\mathrm{RC} 2$ and RC3 do not generate relevant effects on the forces sensed by the dynamometer, therefore they won't be further discussed. On the contrary, some of the instabilities reported in Table 8 clearly lead to unwanted oscillations of the force acting on the shaft. In particular, the two axial instabilities A1 and A2 generate oscillations of $F_{z}$ for corresponding values of $\sigma-f$, while they are not visible at all on a plane perpendicular to the rotational axis $(X Y, x y)$.

Like A1 and A2, the pressure distribution connected with the rotating cavitation-induced instability RC1 generates a fluctuating component on the rotational axis.

However, RC1 also leads to a fluctuating component on the plane XY $(x y)$ at the same operating regimes $(\sigma)$ and frequencies, and with an intensity directly connected to the corresponding energy value.

In order to understand the RC1 effects on the plane $X Y(x y)$, it is useful to analyze the frequency content of the force component $F_{X}$ and $F_{x}$ defined in the fixed frame and in the rotating one, respectively (Figure 14). Let's consider the schematic proposed in Figure 5 and in the following. The frequency energy content of $F_{x y}$ is influenced only by the amplitude and the acting frequency of $\widetilde{F}_{1}$. The pressure distribution due to the presence of cavitation in the form of RC1 generates a rotating force imbalance with a rotational velocity corresponding to the rotational velocity of the phenomenon itself, which is given by the pressure transducer analysis. Moreover, when this pressure distribution interacts with the (static) volute tongue at the impeller exit, it may lead to an oscillating unbalanced force at the same frequency as the phenomenon itself. The above considerations may be summarized as $\omega_{2}=\omega_{1}=2 \pi f_{R C 1}$, therefore

$$
F_{X}=F_{0} \cos \left(\theta_{0}\right)+\left[\widetilde{F}_{1} \cos \left(\theta_{0}\right)+F_{2}\right] \cos \left(\omega_{1} t\right),
$$

which is coherent with the frequency content in Figure 14. Moreover, the corresponding force on the rotating frame is given by

$$
F_{x}=\frac{\widetilde{F}_{1}}{2} \cos \left[\left(\omega_{1}-\Omega\right) t+\theta_{0}\right]+F_{2} \cos \left[\left(\omega_{1}-\Omega\right) t\right]+\frac{\widetilde{F}_{1}}{2} \cos \left[\left(\omega_{1}+\Omega\right) t-\theta_{0}\right]+F_{0} \cos \left(\Omega t-\theta_{0}\right),
$$

where there are only three acting frequencies (as shown in Figure 14, center):

1. $\left(\omega_{1}-\Omega\right) / 2 \pi$, whose intensity depends on the combination of $\widetilde{F}_{1}, F_{2}, \theta_{0}$;

2. $\left(\omega_{1}+\Omega\right) / 2 \pi$;

3. $\Omega / 2 \pi$. 


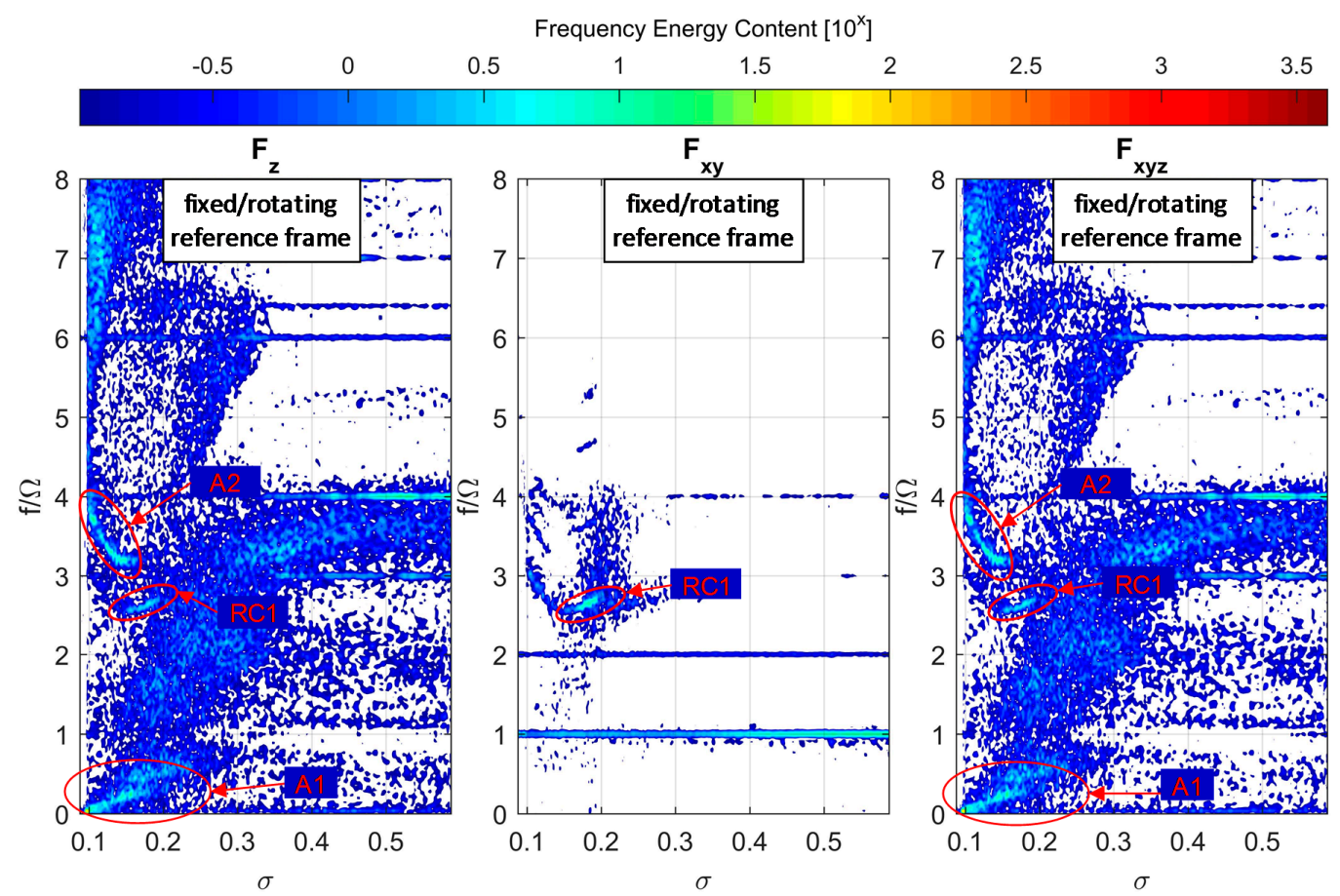

Figure 13. Frequency energy content $\left[\mathrm{N}^{2} \cdot \mathrm{s}\right]$ of the force along the axis $\left(F_{z}\right)$, its perpendicular component $\left(F_{x y}\right)$, and the total force $\left(F_{x y z}\right)$ acting on the dynamometer. Red circled phenomena are summarized in Table 8.

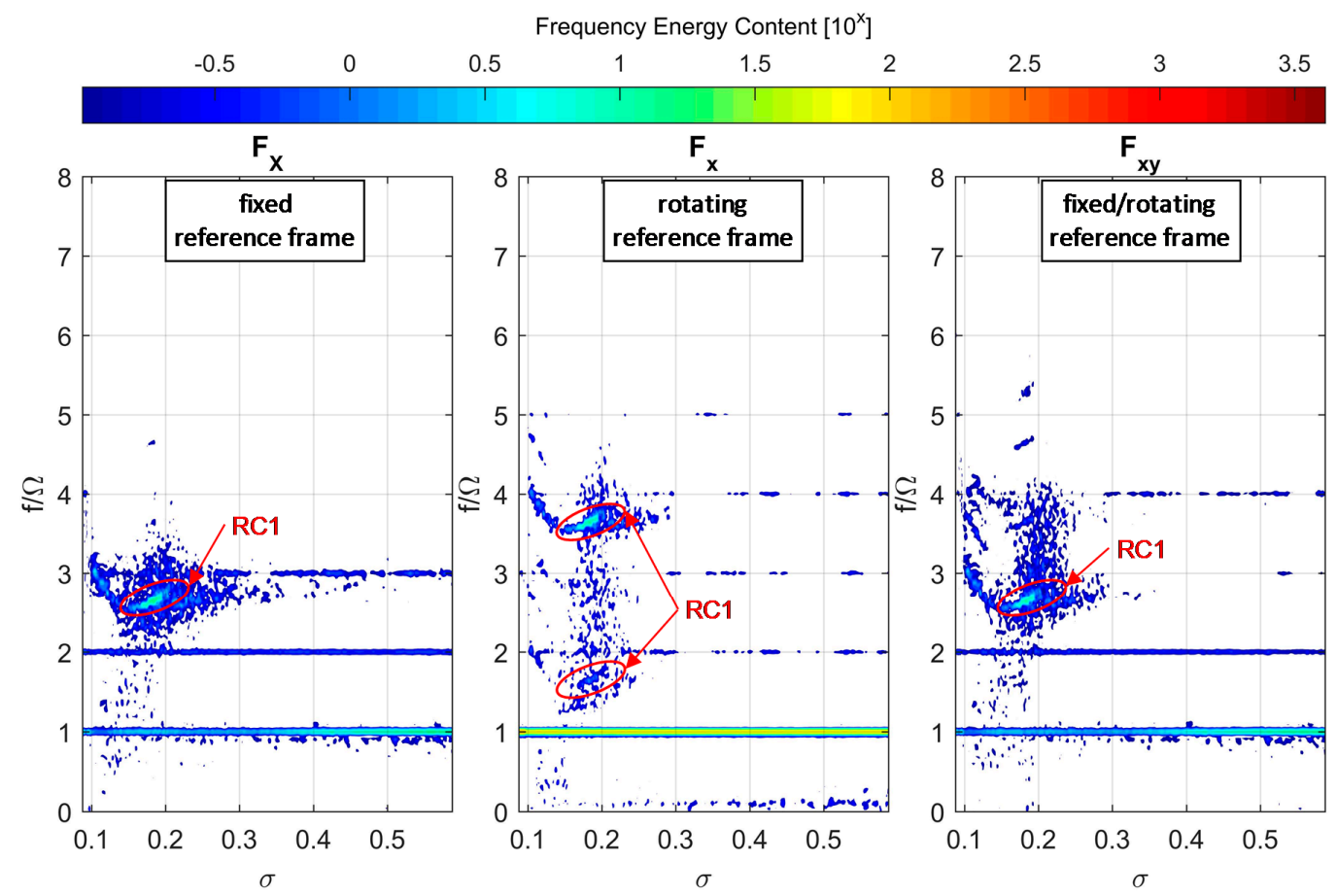

Figure 14. Frequency energy content $\left[\mathrm{N}^{2} \cdot \mathrm{s}\right]$ of the force component along the $\mathrm{X}$-axis in the absolute fixed reference frame $\left(F_{X}\right.$, left), along the $\mathrm{x}$-axis in the rotating reference frame $\left(F_{x}\right)$ and its perpendicular component $\left(F_{x y}\right)$ acting on the dynamometer. Red circled phenomena are summarized in Table 8. 


\section{Conclusions and Future Work}

The experimental characterization of pressure fluctuations on a centrifugal pump for various operating regimes has been here presented and discussed. The paper focuses on the unsteady forces acting on the driving shaft which are triggered by such fluctuations. The contemporary measurement of the unsteady forces and the pressure field on centrifugal pumps constitutes an innovative feature of the present study. Although the paper presents data coming from a specific regime in terms of flow coefficient $\left(\Phi=0.9 \Phi_{D}\right)$, similar behaviors have been detected for analogous phenomena observed during other regimes. In particular, the presence of rotating phenomena may generate fluctuations of the force in the plane perpendicular to the rotational axis as well as on the rotational axis itself. On the perpendicular plane, the frequency content associated with the generated force suggests that the force can be approximated by the sum of a component with a fixed direction and fluctuating intensity together with a purely rotating component. The frequency of both components is the same as for the source phenomenon. A key role in such behavior is most likely played by the single-tongue volute, whose intrinsic asymmetry interacts with the rotating phenomena, leading to force amplitude fluctuations. At $\Phi=\Phi_{D}$ the outlet flow has a nominal zero incidence angle with the tongue, which is most likely the reason why the force triggered by cavitation instabilities is also reduced. This consideration confirms the goodness of the pump design approach described in [26], also from a rotordynamic point of view when cavitation instabilities arise. However, experimental campaigns are needed to better understand the interaction between the presence of cavitation instabilities and forces in presence of different shaped volutes and diffusers. Furthermore, the paper highlights that special attention should be paid when the pump design includes any stator (e.g., a vaned diffuser) especially when the operating conditions may include cavitation.

On the other hand, axial phenomena lead to fluctuations of the force component directed along the rotational axis only.

Interestingly, not all the phenomena measured by the pressure transducers lead to relevant fluctuations of the forces sensed by the dynamometer. Thus, different sensors may underline specific characteristics and effects of the fluid fluctuations. For instance, a strong fluctuation involving a small portion of the blade channels does not necessary lead to a strong fluctuation of the forces on the shaft. On the other hand, phenomena with smaller fluctuation intensities but characterized by large area of interest may involve stronger oscillations of the forces on the shaft which are more dangerous from a structural point of view. Therefore, it is advisable to fully characterize the fluid instabilities and their effects by means of different sensors under the foreseen operating regimes in order to correctly design the system.

The systematic analysis of the signals from the pressure and force sensors with different methods, such as wavelet decomposition, may give some useful insight into the inception and end of each flow pattern although it does not improve the understanding of the fully-developed flow instabilities itself [32]. Therefore, future activities may include such analysis especially when the pump configuration allows for optical access (e.g., an inducer setup at the CPRTF) in order to exactly match the sensors measurements to the cavitation structures.

To date, it is not clear which eventual unstable forces would arise with an inducer ahead of a centrifugal stage (typical in space application) under the presence of cavitation instabilities. However, the present results suggest that the massive presence of such instabilities for an inducer (if compared to a centrifugal stage) may lead to possible dangerous conditions when the inducer is coupled with static elements typical of centrifugal stages (e.g., the volute tongue). Further investigations are needed in order to better clarify these aspects.

Author Contributions: D.V., A.P. and R.H. designed the experiment. D.V., G.P. and R.H. carried out the experimental activities. D.V., G.P., A.P. and R.H. analyzed the data. L.d. supported and inspired the entire project. D.V. wrote the paper. All the authors contributed to writing-review and editing.

Acknowledgments: The present work has been possible thanks to the supports of the European Space Agency along several years. The authors would like to express their great gratitude to Giorgio Saccoccia and to Gianni 
Pellegrini. Special gratitude goes to Lucio Torre that has been a great mainstay of the whole Chemical Propulsion Team for many years and to Kai Su.

Conflicts of Interest: The authors declare no conflict of interest.

\section{Nomenclature}

Latin

A Axial

$A_{f}$ Fluctuation amplitude

$E_{f}$ Fluctuation energy

$\widetilde{F}$ Force intensity fluctuation

$F$ Force

FS Full scale

$N$ Blade number

$Q$ Volume flow rate

$R C$ Rotating cavitation

Re Reynolds

$S$ Power spectrum density

$T$ Temperature

$f$ Frequency

$n$ Flow instability lobes

$p$ Pressure

$r$ Radius

$z$ Axial length, rotating axis

Greek

$\Delta$ Variation

$\Phi$ Flow coefficient

$\Psi$ Head coefficient

$\Omega$ Rotating speed, rotating frequency

$\gamma_{x y}$ Coherence between sensors $x$ and $y$

$v$ Fluid kinematic viscosity

rho Fluid density

$\sigma$ Cavitation number

$\omega$ Force angular frequency

$\varphi$ Cross-spectrum phase

$\theta$ Force initial phase

$\vartheta$ Azimuthal direction

Subscripts

$D$ Design

$N$ Nominal

T Total

c Chamber

in Inlet

$v$ Vapor

$x y z$ Rotating frame

$X Y Z$ Fixed frame

\section{References}

1. Bhattacharyya, A.; Acosta, A.J.; Brennen, C.E.; Caughey, T.K. Rotordynamic Forces in Cavitating Inducers. J. Fluids Eng. 1997, 119, 768. [CrossRef]

2. Bhattacharyya, A. Internal Flows and Force Matrices in Axial Flow Inducers. Ph.D. Thesis, California Institute of Technology, Pasadena, CA, USA, 1994. 
3. Franz, R.J. Experimental Investigation of the Effect of Cavitation on the Rotordynamic Forces on a Whirling Centrifugal Pump Impeller. Ph.D. Thesis, California Institute of Technology, Pasadena, CA, USA, 1989.

4. Rosenmann, W. Experimental investigations of hydrodynamically induced shaft forces with a three bladed inducer. In Proceedings of the ASME Symposium on Cavitation in Fluid Machinery, Chicago, IL, USA, 7-11 November 1965; pp. 172-195.

5. Ehrich, F.; Childs, D. Self-Excited Vibrations in High Performance Turbomachinery. Mech. Eng. 1984, 106, 66-79.

6. Hergt, P.; Krieger, P. Radial Forces in Centrifugal Pumps with Guide Vanes. Proc. Inst. Mech. Eng. 1969, 184, 101-107. [CrossRef]

7. Suzuki, T.; Prunières, R.; Horiguchi, H.; Tsukiya, T.; Taenaka, Y.; Tsujimoto, Y. Measurements of Rotordynamic Forces on an Artificial Heart Pump Impeller. J. Fluids Eng. 2007, 129, 1422-1427. [CrossRef]

8. Brennen, C.E.; Franz, R.; Arndt, N. Effects of Cavitation on Rotordynamic Force Matrices. In Proceedings of the 3rd Conference on Advanced Earth-to-Orbit Propulsion Technology; Richmond, R.J., Wu, S.T., Eds.; NASA: Washington, DC, USA, 1988; pp. 227-239.

9. Hashimoto, T.; Yoshida, M.; Watanabe, M. Experimental Study on Rotating Cavitation of Rocket Propellant Pump Inducers. J. Propuls. Power 1997, 13, 488-494. [CrossRef]

10. Tsujimoto, Y.; Yoshida, Y.; Maekawa, Y.; Watanabe, S.; Hashimoto, T. Observations of Oscillating Cavitation of an Inducer. J. Fluids Eng. 1997, 119, 775-781. [CrossRef]

11. Zoladz, T. Observations on rotating cavitation and cavitation surge from the development of the Fastrac engine turbopump. In Proceedings of the 36th AIAA/ASME/SAE/ASEE Joint Propulsion Conference and Exhibit, Las Vegas, NV, USA, 24-28 July 2000.

12. Tsujimoto, Y.; Semenov, Y. New Types of Cavitation Instabilities in Inducers. In Proceedings of the 4th International Conference on Launcher Technology “Space Launcher Liquid Propulsion”, Liege, Belgium, 3-6 December 2002.

13. Subbaraman, M.; Patton, M. Suppressing Higher-Order Cavitation Phenomena in Axial Inducers. In Proceedings of the 6th International Symposium on Cavitation, Wageningen, The Netherlands, 11-15 September 2006.

14. Kamijo, K.; Yoshida, M.; Tsujimoto, Y. Hydraulic and mechanical performance of LE-7 LOX pump inducer. J. Propuls. Power 1993, 9, 819-826. [CrossRef]

15. Cervone, A.; Bramanti, C.; Rapposelli, E.; Torre, L.; d'Agostino, L. Experimental Characterization of Cavitation Instabilities in a Two-Bladed Axial Inducer. J. Propuls. Power 2006, 22, 1389-1395. [CrossRef]

16. Cervone, A.; Torre, L.; Pasini, A.; d'Agostino, L. Cavitation and Flow Instabilities in a 3- Bladed Axial Inducer Designed by Means of a Reduced Order Analytical Model. In Proceedings of the 7th International Symposium on Cavitation, Ann Arbor, MI, USA, 17-22 August 2009.

17. Torre, L.; Pace, G.; Miloro, P.; Pasini, A.; Cervone, A.; d'Agostino, L. Flow Instabilities on a Three Bladed Axial Inducer at Variable Tip Clearance. In Proceedings of the 13th International Symposium on Transport Phenomena and Dynamics of Rotating Machinery, Honolulu, HI, USA, 4-9 April 2010.

18. Friedrichs, J.; Kosyna, G. Rotating Cavitation in a Centrifugal Pump Impeller of Low Specific Speed. J. Fluids Eng. 2002, 124, 356-362. [CrossRef]

19. Yamamoto, K. Instability in a Cavitating Centrifugal Pump. JSME Int. J. Ser 2 Fluids Eng. Heat Transf. Power Combust. Thermophys. Prop. 1991, 34, 9-17. [CrossRef]

20. Valentini, D.; Pace, G.; Torre, L.; Pasini, A.; d'Agostino, L. Influences of the Operating Conditions on the Rotordynamic Forces Acting on a Three-Bladed Inducer Under Forced Whirl Motion. J. Fluids Eng. 2015, 137, 071304-071304-10. [CrossRef]

21. Valentini, D.; Pace, G.; Pasini, A.; Torre, L.; Hadavandi, R.; d'Agostino, L. Fluid-induced rotordynamic forces on a whirling centrifugal pump. Eur. J. Mech. B/Fluids 2017, 61, 336-345. [CrossRef]

22. Valentini, D.; Pace, G.; Torre, L.; Pasini, A.; d'Agostino, L. Pumping and Suction Performance of a Whirling Inducer. In Proceedings of the Space Propulsion 2014, Cologne, Germany, 19-22 May 2014.

23. Pace, G.; Pasini, A.; Torre, L.; Valentini, D.; d'Agostino, L. Cavitating Pump Rotordynamic Test Facility at ALTA S.p.A.: Upgraded Capabilities of a Unique Test Rig. In Space Propulsion Conference 2012; ESA: Bordeaux, France, May 2012.

24. Pasini, A. Pumping Performance Similarity, Cavitation-Induced Instabilities and Fluid-Induced Rotordynamic Forces in Tapered Inducers. Ph.D. Thesis, University of Pisa, Pisa, Italy, 2010. 
25. d'Agostino, L. Comparison of rotordynamic fluid forces in axial inducers and centrifugal turbopump impellers. IOP Conf. Ser. Mater. Sci. Eng. 2016, 129, 012001. [CrossRef]

26. d'Agostino, L.; Torre, L.; Pasini, A.; Baccarella, D.; Cervone, A.; Milani, A. A Reduced Order Model for Preliminary Design and Performance Prediction of Tapered Inducers: Comparison with Numerical Simulations. In Proceedings of the 44th AIAA/ASME/SAE/ASEE Joint Propulsion Conference \& Exhibit, Hartford, CT, USA, 21-23 July 2008.

27. d'Agostino, L.; Torre, L.; Pasini, A.; Cervone, A. On the Preliminary Design and Noncavitating Performance Prediction of Tapered Axial Inducers. J. Fluids Eng. 2008, 130, 111303:1-111303:8. [CrossRef]

28. Valentini, D.; Pasini, A.; Pace, G.; Torre, L.; d'Agostino, L. Experimental Validation of a Reduced Order for Radial Turbopump Design. In Proceedings of the 49th AIAA/ASME/SAE/ASEE Joint Propulsion Conference, San Jose, CA, USA, 14-17 July 2013.

29. Pace, G.; Valentini, D.; Pasini, A.; Torre, L.; Fu, Y.; d'Agostino, L. Effects of Geometry on Flow Instabilities of Different Three-Bladed Axial Inducers. J. Fluids Eng. 2015, 137, 041304. [CrossRef]

30. Bendat, J.S.; Piersol, A.G. Random Data: Analysis and Measurement Procedures; Wiley Series in Probability and Statistics; John Wiley \& Sons, Inc.: Hoboken, NJ, USA, 2010; ISBN 978-1-118-03242-8.

31. Brennen, C.E. Hydrodynamics of Pumps; Concepts ETI, Inc. and Oxford University Press: Oxford, UK, 1994.

32. Coutier-Delgosha, O.; Dazin, A.; Caignaert, G.; Bois, G. Analysis of Cavitation Instabilities in a Four-Blade Inducer. Int. J. Rotating Mach. 2012, 2012. [CrossRef]

(C) 2018 by the authors. Licensee MDPI, Basel, Switzerland. This article is an open access article distributed under the terms and conditions of the Creative Commons Attribution NonCommercial NoDerivatives (CC BY-NC-ND) license (https://creativecommons.org/licenses/by-nc-nd/4.0/). 